\title{
Population characteristics of golden retriever lifetime study enrollees
}

\author{
Melissa Simpson ${ }^{1^{*}}$ (D), Erin Searfoss ${ }^{1}$, Sharon Albright ${ }^{1}$, Diane E. Brown² ${ }^{2}$ Barbara Wolfe ${ }^{1}$, Nancy K. Clark \\ Susan E. McCann ${ }^{3}$, David Haworth ${ }^{4}$, Mike Guy ${ }^{5}$ and Rod Page ${ }^{6}$
}

\begin{abstract}
Background: Studying cancer and other diseases poses a problem due to their protracted and multifactorial nature. Prospective studies are useful to investigate chronic disease processes since collection of lifestyle information, exposure data and co-incident health issues are collected before the condition manifests. The Golden Retriever Lifetime Study is one of the first prospective studies following privately-owned dogs throughout life to investigate the incidence and risk factors for disease outcomes, especially cancer. Owners of golden retrievers in the contiguous United States volunteered their dogs in early life. Owners and veterinarians complete online questionnaires about health status and lifestyle; dogs undergo a physical examination and collection of biological samples annually. The data presented summarize the initial study visits and the corresponding questionnaires for 3044 dogs in the cohort.

Results: The median age of dogs at enrollment was 14.0 months (interquartile range (IQR): 8-20 months). Approximately half of the population had undergone gonadectomy by their initial study visit. Medical conditions reported at enrollment consisted primarily of integumentary, gastrointestinal and urinary dysfunction. A large majority of the dogs have a record of having received preventive care (vaccines, parasiticides, flea and heartworm prevention) by the time of the initial study visit. Clinical pathology data were unremarkable.
\end{abstract}

Conclusions: This study represents one of the first lifetime observational investigations in veterinary medicine. The population characteristics reported here indicate a healthy cohort of golden retrievers cared for by owners committed to their dogs' health. Data acquired over the study period will provide valuable information about genetic, dietary and environmental risk factors associated with disease in golden retrievers and a framework for future prospective studies in veterinary medicine.

\section{Plain English summary}

Identifying risk factors for cancer is challenging due to the complexity of the disease process and how long it takes for clinical signs associated with disease to become apparent. Longitudinal studies (studies that follow subjects over time with the intention of gaining insight into the development of disease outcomes, ideally study subjects just "live their lives" and are asked to periodically check in to report their experiences) are useful to investigate chronic disease since exposures are collected before the condition manifests. The Golden Retriever

\footnotetext{
* Correspondence: msimpson@morrisanimalfoundation.org

'Morris Animal Foundation, 720 South Colorado Boulevard, Suite 174-A,

Denver, CO 80243, USA

Full list of author information is available at the end of the article
}

Lifetime Study is one of the first longitudinal studies following dogs to investigate the incidence of and risk factors for cancer. Owners of 3044 golden retrievers in the United States volunteered their dogs for this observational cohort. Owners and veterinarians complete online questionnaires about health status and lifestyle of dogs who also undergo a physical examination and collections of biological samples annually. The data presented in this paper are from the dogs' initial study visit. The median age of dogs at enrollment was 14 months; approximately half had undergone spay or neuter surgery. Medical conditions reported at enrollment consisted of skin, digestive and urinary dysfunction and were all minor. A majority of the dogs have a record of receiving preventive care (vaccines, parasiticides, flea, 
and heartworm prevention). Blood work, urinalysis, and fecal parasite test results were largely unremarkable. This study represents one of the first lifetime observational investigations in veterinary medicine. The population characteristics reported here indicate a healthy cohort of golden retrievers cared for by committed owners. This study will provide valuable information about risk factors associated with cancer in golden retrievers and a framework for future prospective studies in veterinary medicine.

\section{Background}

Cancer and other diseases with chronic onset are the result of a complex interplay between environmental and intrinsic host factors. The onset of such diseases may be insidious and are often accompanied by a protracted subclinical prodrome [1]. These attributes make identifying potential etiologies challenging. Identifying putative exposures and characterizing the importance of sensitive period effects, length of exposure, dose, and interactions between exposures is especially challenging with complex and multifactorial diseases. Prospective studies address some of these issues as exposure data are collected in real time and prior clinical manifestations of disease. This feature mitigates the potential for bias that accompanies other study designs and ensures that exposure preceded clinical disease and that both the timing and extent of exposure are collected proximally to when they occurred.

Because of domestic dogs' abbreviated life-span relative to humans, it is more feasible to collect exposure and outcome data over the entire life-course. This attribute makes investigating phenomena such as sensitive period effects and cumulative exposures efficient relative to human studies that are similarly structured. While prospective studies are well-known and regularly employed to study disease etiology and potential risk factors in people [2], to date, large-scale life course veterinary studies have been scarcely reported. Four prospective studies in dogs are currently underway: The Golden Retriever Lifetime Study (GRLS), the 9/11 Medical Surveillance Study of search dogs deployed to the terrorist attack sites [3, 4], Generation Pup, a study following dogs of all breeds from puppy- to adulthood, and Dogslife, a web-based study, which reported data regarding about 4300 Labrador Retrievers in the UK [5-7] and has currently enrolled more than 7000 dogs. The data presented herein are from the GRLS.

The ongoing impetus for the GRLS is to estimate the incidence of and genetic, nutritional, and environmental risk factors for four fatal cancers in golden retrievers: high-grade mast cell tumors, osteosarcoma, hemangiosarcoma, and lymphoma [8-10]. In addition to collecting data about these outcomes, study participants and their veterinarians are asked to report annually about many aspects of the dogs' lives including nutrition, indoor and outdoor environmental exposures, reproductive status and history, preventive health care, behavior and temperament, exercise habits, results of an annual physical examination, clinical pathology laboratory results, and all major health events. All clinical pathology data are collected by a single reference laboratory. All clinical pathology results were shared with owners through their personal veterinarians and, when needed (Eg: positive heartworm results), appropriate treatments were pursued as per the normal veterinary/client/patient relationship.

The purpose of this report is to present descriptive characteristics of a cohort of 3044 golden retrievers at enrollment.

\section{Methods}

\section{Study population}

The data presented in this study were collected on 3044 golden retrievers enrolled in GRLS. Details about the structure of the study, recruitment and enrollment process and data collection have been previously published [11]. Briefly, we recruited 3044 privately-owned dogs living in the contiguous United States that were between six months and two years of age at the time of entry into the study. Knowledge of prior pedigree for at least two generations was required. Both the owner and veterinarian gave informed consent to participate and committed to the requirements of the study which include annual internet-based questionnaires (including both structured and free text response options) for both the owner and veterinarian, complete veterinary physical examinations, and biological sample collection (whole blood and DNA, serum, urine, feces, hair, and toenails).

In addition, at the time of a diagnosis of any malignancy (not solely the four primary outcomes), another set of biological samples are obtained in addition to a physical exam report, diagnostic laboratory reports, and histopathology samples, when appropriate.

Recruitment was started in 2012 and all requirements outlined above were completed on 3044 (100\%) dogs in March 2015; our target was 3000 dogs and enrollment closed when that goal was met. The additional 44 dogs were enrolled because they completed enrollment requirements before enrollment officially closed. Body condition score was reported by veterinarians using the Purina ${ }^{\circ}$ nine point scale [12].

The study protocol and participation requirements were reviewed and approved by Morris Animal Foundation's appointed Animal Welfare Advisory Board and informed consent was obtained from all owners and veterinarians prior to enrollment. 


\section{Analysis}

The data presented are from the owner questionnaire, the initial, qualifying clinical visit captured in the veterinarian questionnaire, and clinical pathology results obtained at the time of enrollment. Because the data are descriptive, no formal statistical tests were performed. To ascertain veterinarian-reported medical conditions, we asked the question by body system, "Indicate if the dog has been diagnosed with a given condition in the past 12 months." To account for interval censoring in the reporting of medical conditions when calculating incidence density, we estimated that all diagnoses occurred 6 months prior to the initial clinical visit for dogs 6 months or older at the time of the visit. For dogs younger than 6 months at the time of their visit, we used the assumption that all diagnoses occurred at age (in months)/2. All tables and figures were generated using commercially available statistical software. ${ }^{1}$

\section{Results}

We defined five regions of the contiguous U. S. to direct recruitment efforts: Northeast, South, Midwest, Mountain, and Pacific (Fig. 1). In addition, Fig. 1 indicates the general quantitative range of dogs enrolled by state. California had the largest number enrolled $(n=278)$ while Colorado had the largest enrolled per human capita $(n=255 ; 5$ dogs/ 100,000 people) and most states have fewer than 70 dogs enrolled.

Table 1 contains basic descriptive information about the study dogs at the initial clinical visit. The median age of study dogs at the initial clinical visit was 14.0 months (IQR 8.3-20.3). Overall, 578 (19\%) owners reported having pet health insurance. Ninety five percent of the dogs in this study were acquired from breeders. All but 3\% of study dogs sleep in the owner's house and $84 \%$ of owners report that the primary job of their dog is to be a pet or companion rather than primarily a working or service dog. Of the 1137 (37\%) dogs who had undergone gonadectomy prior to their first study visit, the majority (97\%) were elective rather than the result of a medical or behavioral indication. Among male dogs, cryptorchid testicles were the most common nonelective indication for gonadectomy ( 85 dogs, $16 \%$ of neutered males). Four female dogs underwent gonadectomy for medical indications, including three dogs with cystitis and one with metritis.

Owner-reported environmental and lifestyle exposures are shown in Table 2. Most dogs (83\%) receive both ectoparasite (flea and tick) and heartworm prevention, $15 \%$ only receive heartworm prevention, $5 \%$ only receive ectoparasite prevention, and $6 \%$ do not receive either medication. Sixty four percent of Americans as a whole report purchasing flea prevention products [13]. Of the dogs who receive preventive medications, 1805 (59\%) receive ectoparasite preventive topically and 2454 (81\%) receive heartworm prevention orally. Almost 100\% of dog owners report giving their dogs treated (Eg: municipal water) or filtered well water as their source of drinking water (these water sources are considered safe for human consumption) and that they live in a suburban environment (60\%); urban living conditions are the least frequently reported (10\%). A small portion of participating dogs $(6 \%)$ are exposed to secondhand

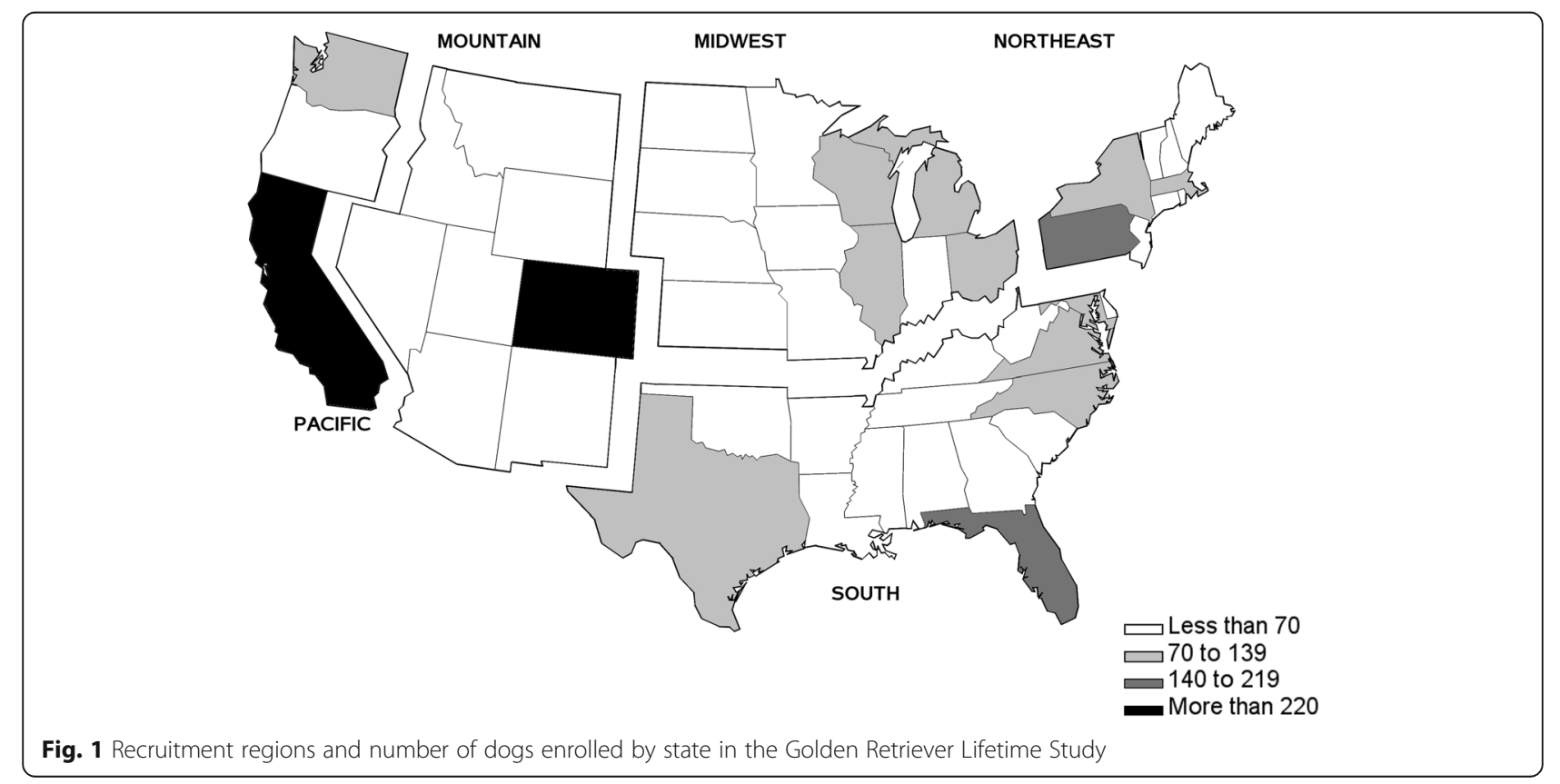


Table 1 Descriptive characteristics of 3044 Golden Retriever Dogs participating in the Golden Retriever Lifetime Study at their initial clinical study visit

\begin{tabular}{|c|c|c|c|c|c|}
\hline \multirow[t]{3}{*}{ Characteristic } & \multicolumn{5}{|l|}{ Region } \\
\hline & Northeast & Midwest & South & Mountain & Pacific \\
\hline & $n=635$ & $n=722$ & $n=841$ & $n=413$ & $n=433$ \\
\hline \multicolumn{6}{|l|}{ Sex } \\
\hline$n(\%)$ spayed female & $118(19)$ & $151(21)$ & $182(22)$ & $86(21)$ & $101(23)$ \\
\hline$n(\%)$ intact female & $203(32)$ & $201(28)$ & $231(27)$ & $109(26)$ & $122(28)$ \\
\hline$n(\%)$ neutered male & $88(14)$ & $127(18)$ & $132(16)$ & $87(21)$ & $82(19)$ \\
\hline$n(\%)$ intact male & $226(36)$ & $243(34)$ & $296(35)$ & $131(32)$ & $128(30)$ \\
\hline Median age at spay/neuter in months ( \pm IQR) & $6.8(6.1-10.3)$ & $6.4(5.5-8.4)$ & $6.7(5.6-9.4)$ & $6.6(5.6-9.1)$ & $7.1(5.9-10.8)$ \\
\hline$n(\%)$ reporting elective spay/neuter & $199(97)$ & $273(98)$ & $309(98)$ & $165(95)$ & $174(95)$ \\
\hline$n(\%)$ Registered with the American Kennel Club & $547(86)$ & $603(84)$ & $741(88)$ & $321(78)$ & $367(85)$ \\
\hline$n(\%)$ with pet health insurance & $150(24)$ & $79(11)$ & $148(18)$ & $91(22)$ & $110(25)$ \\
\hline $\begin{array}{l}n(\%) \text { acquired from a breeder (includes bred by } \\
\text { current owner) }\end{array}$ & $612(96)$ & $688(95)$ & $791(94)$ & $374(91)$ & $418(97)$ \\
\hline$n(\%)$ sleeping in the house & $629(99)$ & $697(97)$ & $826(98)$ & $403(98)$ & $411(95)$ \\
\hline$n(\%)$ primary activity is companion or pet & $520(83)$ & $573(80)$ & $697(84)$ & $360(88)$ & $371(87)$ \\
\hline
\end{tabular}

smoke and exposure time ranges from $1 \mathrm{~h}$ per day to $24 \mathrm{~h}$ per day. Most owners feed a commercial diet (85\%); among owners who report feeding a homeprepared diet, 257 (55\% of home-prepared feeders, 8\% of the entire cohort) report feeding a diet that is at least partially raw.

The results of veterinary physical examination are shown in Table 3 . The median body condition score (BCS) was 5 (IQR 5-6), 868 (29\%) dogs had a BCS of $5.5 / 9$ (i.e. overweight) or higher. The median height at the withers for all sexes combined was $57.2 \mathrm{~cm}$ (IQR: 54.6-61.0 cm); median weight for all sexes combined was $27.8 \mathrm{~kg}$ (IQR: 24.4-31.6 kg). Overall, 1822 (59\%) of dogs had a normal physical exam. The most common abnormalities noted on physical exam at the initial clinical visit were dental tartar $(n=336,11 \%)$, otitis externa ( $n=309,10 \%)$, and skin conditions $(n=259,9 \%)$.

Table 4 shows prevalence and incidence density of diagnoses reported in the 12 months preceding the initial clinical visit by body system and the most commonly reported diagnosis with each system. Study dogs accrued 15,492.5 dog months (mean of 5 months per dog) at risk when calculating incidence density of veterinary-reported conditions. Problems of the integumentary system were the most commonly reported. Otitis externa was the most common diagnosis, followed by enteritis.

Eighty four percent $(n=2549)$ of dogs received at least one dose of American Animal Hospital Association recommended core vaccines (rabies, parvovirus, distemper, and adenovirus) [14]; 90\% of dogs received at least one vaccination against rabies virus, and 72 (2\%) dogs received both a one-year and a three-year rabies vaccine during the preceding 12 months. The most common non-core vaccines reported were four-way leptospirosis (47\%, $n=1444)$, and intranasal Bordetella (36\%, $n=1106)$. Eighteen percent $(n=549)$ of dogs received a vaccine against Borrelia burgdorferi. Two hundred ninety nine (10\%) dogs did not have a record of having received a vaccination on or prior to the initial clinical study visit.

As part of the annual study visit, a serum chemistry panel, total $\mathrm{T} 4$, heartworm antigen test, complete blood count (CBC), urinalysis, and fecal pathogens analysis were performed. Forty percent $(n=1198)$ of dogs had all values included on a $\mathrm{CBC}$ within the normal limits established by the reference laboratory. On serum chemistry, 1828 (60\%) of dogs had all values within reference ranges. Quantitative urinalysis results were $100 \%$ within reference laboratory normal limits; eleven dogs $(<1 \%)$ tested positive for heartworm antigen; 64 (2\%) dogs had low total T4 (minimum value: $0.49 \mu \mathrm{g} / \mathrm{dl}$; reference range: $0.8-3.5 \mu \mathrm{g} / \mathrm{dl})$, and $282(9 \%)$ dogs had at least one parasite on fecal flotation. A list of the clinical pathology tests, normal ranges, and units can be found in Additional file 1: Table S1.

\section{Discussion}

This cohort of golden retrievers will be followed through their life-course to estimate the incidence of cancer and other major diseases or disorders and investigate the risk factors for these conditions. As the study progresses, this population of dogs will receive care from their primary and referral veterinarians who will systematically contribute data to the study. This attribute will allow for collection of data from a population of dogs whose owners pursue a wide spectrum of veterinary care. 
Table 2 Owner-reported environmental and lifestyle exposures among 3044 Golden Retriever Dogs at the time of first owner questionnaire

\begin{tabular}{|c|c|c|}
\hline Characteristic & $n$ & $\%$ \\
\hline Flea prevention & 2389 & 79 \\
\hline Seasonal prevention & 598 & 20 \\
\hline Year-round prevention & 1791 & 59 \\
\hline Heartworm prevention & 2702 & 89 \\
\hline Seasonal prevention & 611 & 20 \\
\hline Year-round prevention & 2091 & 69 \\
\hline \multicolumn{3}{|l|}{ Drinking water source } \\
\hline Municipal & 809 & 27 \\
\hline Well, treated or filtered & 2211 & 73 \\
\hline Well, untreated & 24 & 1 \\
\hline Swims at least weekly & 1200 & 39 \\
\hline Drink or eat from a plastic bowl & 683 & 22 \\
\hline Eat feces & 777 & 26 \\
\hline \multicolumn{3}{|l|}{ Owner reported physical activity level } \\
\hline Little or none & 28 & 1 \\
\hline Moderate & 1469 & 48 \\
\hline Very active & 1547 & 51 \\
\hline \multicolumn{3}{|c|}{ Owner reported insecticide or herbicide treatment } \\
\hline In the house & 662 & 22 \\
\hline In the yard & 1136 & 37 \\
\hline \multicolumn{3}{|l|}{ Owner reported grass eating behavior } \\
\hline Frequent & 823 & 27 \\
\hline Infrequent & 1701 & 56 \\
\hline Never & 519 & 17 \\
\hline \multicolumn{3}{|l|}{ Type of residence } \\
\hline Urban & 317 & 10 \\
\hline Suburban & 1837 & 60 \\
\hline Rural & 890 & 29 \\
\hline Second hand smoke exposure & 185 & 6 \\
\hline Median hours exposed per day $( \pm \mid \mathrm{QR})$ & 3.5 & 4.1 \\
\hline \multicolumn{3}{|l|}{ Type of dog food } \\
\hline Commercially prepared & 2580 & 85 \\
\hline Home prepared & 392 & 13 \\
\hline Both commercially and home prepared & 72 & 2 \\
\hline
\end{tabular}

A variety of owner-reported exposures and lifestyles will allow investigation of the association between these exposures and important health outcomes. While there is potential for misclassification of exposure with ownerreported data [15], annual collection of the same information over time will allow us to study trends. Future nested studies using the data and samples collected on this cohort will examine a multitude of important associations, including nutrition. In addition, as novel biomarkers of health and disease are developed, we will utilize prospectively collected samples to study these biomarkers as a way to potentially identify disease earlier and give insight into etiology of disease.

At the time of the initial clinical study visit, study dogs were largely healthy with skin and gastrointestinal problems being the most commonly reported veterinary diagnoses. These conditions are commonly diagnosed in young dogs $[16,17]$. Clinically significant abnormalities on routine laboratory tests were rare, and all dogs were free from chronic or life-limiting disease at the time of initial physical exam, as this was an exclusion criterion. A common abnormality found was low total T4 on the serum chemistry panel. As the study progresses, these abnormalities and all major illnesses reported by veterinarians will be adjudicated by a panel of subject matter experts in order to ensure standardized disease definitions.

About half of the dogs were gonadectomized at the initial clinical study visit, compared to the national average of $85 \%$ [13]. The importance of reproductive hormones in the health and disease of these dogs will be investigated as the study progresses. The median height and weight parameters for participating dogs were within breed standard regardless of gender or reproductive status (Males: $58-61 \mathrm{~cm}$ at the withers and 29.5$34 \mathrm{~kg}$, Females: $55-57 \mathrm{~cm}$ at the withers and 25 to $29.5 \mathrm{~kg}$ ) [18]. As the study progresses, it will be important to compare mature height and weight between intact and gonadectomized dogs as well as the effect of age at gonadectomy on these parameters. In addition to height and weight, reproductive hormone exposure [number of estrous cycles (females) or years (males) prior to gonadectomy] will be studied in the context of health and disease outcomes. This will have important practical implications for practicing veterinarians when counseling their clients about if or when to get their dogs reproductively altered.

This study was designed to operate until 500 combined cases of the four primary outcomes (high grade mast cell tumor, hemangiosarcoma, osteosarcoma, and lymphoma) occur. We estimated that this number of cases will take about ten to twelve years to develop. During the intervening years we will continue to collect owner- and veterinarian-reported data and biological samples; the pairing of clinical data and samples will facilitate many novel etiologic investigations into many important clinical outcomes. While we designed the study to have sufficient power to study the four primary cancer outcomes, we will collect information about many disease outcomes.

While this study has the advantage that dogs were recruited from a general population with enrollment stratified by region and gender, we still have a highly 
Table 3 Results from physical examination on 3044 Golden Retriever dogs at their initial clinical study visit

\begin{tabular}{|c|c|c|c|c|}
\hline \multirow[t]{3}{*}{ Characteristic } & \multicolumn{4}{|l|}{ Sex } \\
\hline & Male intact & Male neutered & Female intact & Female spayed \\
\hline & $n=1024$ & $n=516$ & $n=866$ & $n=638$ \\
\hline Median Purina ${ }^{\oplus}$ Body Condition Score $( \pm \mid \mathrm{QR})$ & $5(5-5)$ & $5(5-6)$ & $5(5-5)$ & $5(5-6)$ \\
\hline Median weight in $\mathrm{Kg}( \pm \mathrm{QR})$ & $29.1(25.5-32.3)$ & $32.5(29.1-35.9)$ & $24.8(21.7-27.4)$ & $27.5(24.8-30.8)$ \\
\hline Median height at withers in $\mathrm{cm}( \pm \mid \mathrm{QR})$ & $58.4(55.9-61.0)$ & $61.0(58.4-63.5)$ & $54.6(53.0-57.2)$ & $57.2(54.6-60.1)$ \\
\hline Median age in months at exam $( \pm \mid \mathrm{QR})$ & $11.0(7.3-17.1)$ & 18.5(13.7-23.3) & $11.1(7.4-17.6)$ & $16.9(12.2-22.5$ \\
\hline $\mathrm{n}(\%)$ with a normal physical exam & $633(62)$ & $268(52)$ & $556(64)$ & $365(57)$ \\
\hline
\end{tabular}

selected population of dogs and the applicability of study results to other canine populations remains to be determined. Owners are encouraged to pursue diagnostic and treatment for all conditions after careful consultation with their primary veterinarian so we do not expect that participation in the study will influence diagnosis or course of clinical disease but the possibility of participant bias is remains. A similar study in Labrador retriever dogs may provide a means to compare results and assess the degree to which these data can be applied to other populations [6].

Retaining study dogs and maintaining owner engagement will be a crucial element in ensuring that data are representative of the original cohort. Ongoing retention efforts include encouraging participant engagement

Table 4 Prevalence and incidence density of diagnoses by body system and the most common diagnosis within systems in a cohort of 3044 Golden Retriever dogs during the 12 months preceding their initial clinical study visit

\begin{tabular}{lll}
\hline Body system & $\begin{array}{l}\text { Prevalence } \\
\text { (diagnoses/ } \\
3044 \text { dogs } \\
\text { enrolled) }\end{array}$ & $\begin{array}{l}\text { Incidence } \\
\text { density } \\
\text { (new diagnoses } \\
\text { per 1000 } \\
\text { dog-months) }\end{array}$ \\
\hline Integumentary $(n=943)$ & 0.31 & 61.51 \\
Otitis externa $(n=561)$ & 0.18 & 36.21 \\
Gastrointestinal $(n=578)$ & 0.19 & 36.79 \\
Enteritis (acute and/or self-limiting) & 0.12 & 23.37 \\
( $n=365)$ & & \\
Urinary $(n=274)$ & 0.09 & 17.94 \\
Bladder infection/Cystitis $(n=273)$ & 0.09 & 17.69 \\
Musculoskeletal $(n=213)$ & 0.07 & 14.72 \\
Pain or lameness $(n=59)$ & 0.02 & 6.45 \\
Ophthalmic $(n=161)$ & 0.05 & 10.39 \\
Conjunctivitis $(n=121)$ & 0.04 & 7.75 \\
Reproductive $(n=91)$ & 0.03 & 6.52 \\
Cryptorchid $($ unilateral or bilateral)( $n=61)$ & 0.02 & 4.84 \\
Neurologic $(n=30)$ & 0.01 & 1.10 \\
Epilepsy $(n=7)$ & 0.00 & 0.45 \\
\hline
\end{tabular}

through social media, special events to recognize participants and dogs, and technical support. At the time of submission of this manuscript, $28(<1 \%)$ dogs had been withdrawn from the study, 51 had died of various conditions including cancer, and $84 \%$ of the cohort is fully compliant with study requirements. This compliance rate is high compared to cohort studies involving a similar scope and time frame in humans $[19,20]$. Factors reported at enrollment that were associated with oneyear compliance in our cohort included sleeping in the owners' bedroom, regular grooming, and current with core vaccinations [21].

\section{Conclusion}

The value of long-term prospective studies in the advancement of human and veterinary health is difficult to understate; many important disease associations have arisen from those studies and they continue to inform treatment and prevention recommendations and public health policy [22-24]. The need for similar data in companion animals is equally important and this study is designed to address some of the gaps in evidencebased recommendations for veterinary medicine.

\section{Endnotes}

${ }^{1}$ SAS version 9.4 (SAS Institute Inc., Cary, NC, USA)

\section{Additional file}

Additional file 1: Table S1. List of clinical pathology tests routinely performed, the reference ranges and units. (DOCX $14 \mathrm{~kb}$ )

\section{Acknowledgements}

We would like to thank the participating dog owners, study veterinarians and golden retrievers for their ongoing contributions and support. In addition, we thank the staff at Morris Animal Foundation for their hard work and support of this study.

\section{Funding}

The Golden Retriever Lifetime study and this manuscript were made possible through financial support provided by the Morris Family Foundation, Blue Buffalo Cancer Research Foundation, Petco Foundation, Zoetis, Antech Inc., the Golden Retriever Foundation, the Hadley and Marion Stuart Foundation, Mars veterinary, generous private donors, and the Flint Animal Cancer Center, Colorado State University. The study is managed and executed by 
Morris Animal Foundation and several co-authors are employed by Morris Animal Foundation, however, final scientific content is written and approved independently of the entities that funded the study. All other funding sources did not have any involvement in the study design, data analysis and interpretation, or writing and publication of the manuscript.

\section{Availability of data and materials}

The data sets used and/or analyzed during the current study are available from the corresponding author on reasonable request.

\section{Authors' contributions}

Melissa Simpson analyzed the data and wrote the manuscript. Erin Searfoss, Sharon Albright, Barbara Wolfe, Mike Guy, Diane Brown, Susan McCann, Nancy Kay Clark, and David Haworth made substantial contributions to data collection and editing the manuscript. Rod Page is the principal investigator and made substantial contributions to study design, data collection and interpretation, and editing the manuscript. All authors read and approved the final manuscript.

\section{Ethics approval}

All owners and veterinarians participating in the Golden Retriever Lifetime Study provided informed consent to study their dogs and publish findings resulting from the study. The study protocol was reviewed and approved my Morris Animal Foundation's Animal Welfare Advisory Board.

\section{Consent for publication}

not applicable.

\section{Competing interests}

The authors declare that they have no competing interests.

\section{Publisher's Note}

Springer Nature remains neutral with regard to jurisdictional claims in published maps and institutional affiliations.

\section{Author details}

${ }^{1}$ Morris Animal Foundation, 720 South Colorado Boulevard, Suite 174-A, Denver, CO 80243, USA. ${ }^{2}$ American Kennel Club Canine Health Foundation, P.O. Box 900061, Raleigh, NC 27675, USA. ${ }^{3}$ Roswell Park Cancer Institute, Elm and Carlton Streets, Buffalo, NY 14263, USA. ${ }^{4}$ PetSmart Charities, 19601 North 27th Avenue, Phoenix, AZ 85027, USA. 5Jaguar Pharmaceuticals, 201 Mission Street, Suite 2375, San Francisco, CA 94105, USA. ${ }^{6}$ Colorado State University, College of Veterinary Medicine and Biomedical Sciences, Fort Collins, CO 80523-1620, USA.

Received: 18 July 2017 Accepted: 1 November 2017

Published online: 15 November 2017

\section{References}

1. Hlatky L, Hahnfeldt P. Beyond the cancer cell: progression-level determinants highlight the multiscale nature of carcinogenesis risk. Cancer Res. 2014;74:659-64.

2. Mahmood SS, Levy D, Vasan RS, Wang TJ. The Framingham heart study and the epidemiology of cardiovascular disease: a historical perspective. Lancet. 2014;383:999-1008.

3. Otto $C M$, Downend $A B$, Moore $G E$, et al. Medical surveillance of search dogs deployed to the world trade center and pentagon: 2001-2006. J Environ Health. 2010;73:12-21.

4. Fitzgerald SD, Rumbeiha WK, Emmett Braselton W, Downend AB, Otto CM. Pathology and toxicology findings for search-and-rescue dogs deployed to the September 11, 2001, terrorist attack sites: initial five-year surveillance. J Vet Diagn Investig. 2008;20:477-84.

5. Clements DN, Handel IG, Rose E, et al. Dogslife: a web-based longitudinal study of Labrador retriever health in the UK. BMC Vet Res. 2013;9:13.

6. Pugh CA, Bronsvoort BM, Handel IG, Summers KM, Clements DN. Dogslife: a cohort study of Labrador retrievers in the UK. Prev Vet Med. 2015;122:426-35.

7. Pugh CA, Summers KM, Bronsvoort BM, Handel IG, Clements DN. Validity of internet-based longitudinal study data: the elephant in the virtual room. J Med Internet Res. 2015;17:e96.
8. Boerkamp KM, Teske E, Boon LR, Grinwis GC, van den Bossche L, Rutteman GR. Estimated incidence rate and distribution of tumours in 4,653 cases of archival submissions derived from the Dutch golden retriever population. BMC Vet Res. 2014;10:34.

9. Edwards DS, Henley WE, Harding EF, Dobson JM, Wood JL. Breed incidence of lymphoma in a UK population of insured dogs. Vet Comp Oncol. 2003;1:200-6.

10. Fleming JM, Creevy KE, Promislow DE. Mortality in north american dogs from 1984 to 2004: an investigation into age-, size-, and breed-related causes of death. J Vet Intern Med. 2011;25:187-98.

11. Guy MK, Page RL, Jensen WA, et al. The golden retriever lifetime study: establishing an observational cohort study with translational relevance for human health. Philos Trans R Soc Lond Ser B Biol Sci 2015;370:1-10.

12. Mawby DI, Bartges JW, d'Avignon A, Laflamme DP, Moyers TD, Cottrell T. Comparison of various methods for estimating body fat in dogs. J Am Anim Hosp Assoc. 2004;40:109-14.

13. Association APP. APPA National pet Owners Survey. Greewich, CT: American Pet Products Association, Inc.; 2017.

14. Paul MA, Carmichael LE, Childers H, et al. 2006 AAHA canine vaccine guidelines. J Am Anim Hosp Assoc. 2006;42:80-9.

15. Newell SA, Girgis A, Sanson-Fisher RW, Savolainen NJ. The accuracy of selfreported health behaviors and risk factors relating to cancer and cardiovascular disease in the general population: a critical review. Am J Prev Med. 1999;17:211-29.

16. Freeman LM, Abood SK, Fascetti AJ, et al. Disease prevalence among dogs and cats in the United States and Australia and proportions of dogs and cats that receive therapeutic diets or dietary supplements. J Am Vet Med Assoc. 2006;229:531-4

17. Lund EM, Armstrong PJ, Kirk CA, Kolar LM, Klausner JS. Health status and population characteristics of dogs and cats examined at private veterinary practices in the United States. J Am Vet Med Assoc. 1999;214:1336-41.

18. Club AK. Offical Standard for the Golden Retriever. In. https://www.grca.org/ about-the-breed/akc-breed-standard/ American Kennel Club, 1990:2.

19. Toledano MB, Smith RB, Brook JP, Douglass M, Elliott P. How to establish and follow up a large prospective cohort study in the 21 st century-Lessons from UK COSMOS. PLoS One. 2015;10:e0131521.

20. Galea S, Tracy M. Participation rates in epidemiologic studies. Ann Epidemiol. 2007:17:643-53.

21. Ruple A, Jones M, Simpson M, Searfoss E, Wolfe B, Page R. The golden retriever lifetime study: assessing factors affecting owner compliace after the first year of enrollment. Orlando, FL: Veterinary Cancer Society Annual Conference; 2016.

22. Black N. Why we need observational studies to evaluate the effectiveness of health care.

23. Chen PML, James SH, James ML, David RW, Richard PM, Chen Jieming. Socioeconomic Factors, Health Behaviors, and Mortality: Results From a Nationally Representative Prospective Study of US Adults.

24. Pugh CA, Bronsvoort BM, Handel IG, Summers KM, Clements DN. What can cohort studies in the dog tell us? Canine Genet Epidemiol. 2014;1:5.

\section{Submit your next manuscript to BioMed Central and we will help you at every step:}

- We accept pre-submission inquiries

- Our selector tool helps you to find the most relevant journal

- We provide round the clock customer support

- Convenient online submission

- Thorough peer review

- Inclusion in PubMed and all major indexing services

- Maximum visibility for your research

Submit your manuscript at www.biomedcentral.com/submit
) Biomed Central 